This is an Open Access article distributed under the terms of the Creative Commons Attribution 4.0 International License which permits unrestricted non-commercial use, distribution, and reproduction in any medium, provided the original work is properly cited.

\title{
EFFECTIVENESS OF SOCIAL SKILLS TRAINING (SST) BASED ON COMPUTER AND MANUAL FOR IMPROVING SOCIALIZATION AND SOCIAL FUNCTION OF SCIZOFRENIA PATIENTS: SYSTEMATIC REVIEW
}

\author{
SITI KOTIJAH ${ }^{1}$, ASEF WILDAN MUNFADLILA ${ }^{2}$
}

Bina Sehat PPNI health science institute Mojokertoregency East Java Indonesia

Email : khodijahsiti105@gmail.com, acheif90@gmail.com.

\section{ABSTRACT}

\section{Keywords}

Background: Schizophrenia is part of a psychotic disorder characterized by a loss of understanding of reality and a loss of insight that can be found in symptoms of severe mental disorders such as hallucinations, delusions, chaotic behavior, and disorganized speech, as well as negative symptoms that cause social damage. Psychosocial interventions such as Social Skills Training (SST) is one of the efforts that can be given to schizophrenic patients to increase patient socialization and communication skills so that patients can adapt to their environment.

Research Objectives: To determine the effectiveness of computer-based and manual-based Social Skills Training (SST) on improving the socialization and social function of schizophrenic patients.

Data Sources: Sources of data obtained from Pubmed, ScienceDirect, and Ebsco

Inclusion Criteria: 1) research studies using the RCT approach, 2) reviews in schizophrenic patients in the adolescent-adult age range (17 years- 80 years), 3 ) studies with social therapy interventions, skills training combined social cognitive therapy treatments, both as independent interventions or in combination with other interventions

Appraisal studies and synthesis methods: Appraisal studies use the critical appraisal skills program (CASP), and the synthesis method uses PICO modifications.

Results: Social skill training (SST) has a significant influence in overcoming the improvement of socialization and social functions

Conclusions and Implications of Results: Social skill training (SST) provides a significant impacton improving socialization and social functions so that it can be applied as an effort to overcome social damage.
Social Skills

Training

(SST),

Social skill

training

(SST),

Computer and Manual. 


\section{INTRODUCTION}

Schizophrenia is a severe mental disorder that can affect the thoughts, feelings, and behavior of individuals. Schizophrenia is part of a psychotic disorder that is mainly characterized by a loss of understanding of reality and a loss of insight (Mudge et al., 2008). Schizophrenia is a complex mental illness and is one of the conditions associated with the highest economic burden of health care in the world (Mudge et al., 2008). Although many studies have shown the effectiveness of antipsychotic drugs in controlling positive symptoms, it is difficult to effectively eliminate negative symptoms and cognitive disorders that correlate with social functioning (Witthöft \& Jasper, 2015). Therefore treatment with drugs alone is not enough to promote rehabilitation of patients with schizophrenia. Care for this population must combine treatment with psychosocial interventions (Weisz, Sandler, Durlak, \& Anton, 2005).

Schizophrenia disorders have a lifetime prevalence of $0.3-0.7 \%$ (Saito et al., 1998). The global prevalence rate of schizophrenics in 2016 according to WHO there is around 21 million affected by schizophrenia. In Indonesia, with a variety of biological, psychological and social factors with a diversity of the population, the number of cases of mental disorders continues to increase which has an impact on increasing the burden on the country and decreasing human productivity in the long run. Riskesdas 2013 data shows the prevalence of heavy mental disorders such as schizophrenia reaches around 400,000 people or as many as 1.7 per 1,000 population. Patients with schizophrenia have a 2.6 -fold mortality rate of 2.6 causes compared to the population without schizophrenia(Chavushyan, Hovsepyan, \& Boyajyan, 2013).

In psychosis disorders, including schizophrenia can be found symptoms of severe mental disorders such as hallucinations, delusions, chaotic behavior, and chaotic speech, as well as negative symptoms that cause social damage(Tényi,
Herold, Szili, \& Trixler, 2002). Social damage is a core problem of schizophrenia which is a major barrier to recovery. Some psychotic symptoms are partially improved by the use of drugs, but the route to recovery is hampered by social disorders because existing social skills interventions tend to lack access, high burden and low adherence, so that effective alternative strategies are needed in resolving them(Verdoux \& Van Os, 2002).

Efforts can be made to prevent or minimize complications arising in schizophrenics with psychosocial care modality therapy, namely Social Skills Training (SST). Social Skills Training (SST) is a separate intervention focus on two components that aim to improve the patient's social functioning and is one of the treatments of choice in schizophrenic spectrum disorders. Research on social cognition in schizophrenia has received increasing attention over the past ten years because it has proven to be substantially a social cognitive deficit in social functioning(Green, Olivier, Crawley, Penn, \& Silverstein, 2005). Social cognition is defined as a mental operation that supports perceptions, interpretations, and responses during social interactions; including the intentions, dispositions, and behavior of others(Burns, 2006).

\section{METHOD}

\section{Design}

The design carried out in this paper is a systematic review. The Effect of Social Skills Training (SST) on improving the socialization ability of schizophrenic patients will be reviewed, including how to take samples and measured variables. Database search and article screening are conducted independently by reviewers by following the requirements in meeting the inclusion criteria. 
2. Criteria for inclusion and exclusion of research respondents

Inclusion criteria respondents

1) Research type

The type of research examined in this study is the result of the publication of all types of quantitative research by providing psychosocial therapy to the sample with a randomized control trial (RCT) and Quasiexperimental approach.

2) Characteristics of research respondents In this Systematic review study was centered in accordance with the results of the study with the criteria for respondents of schizophrenia patients with adolescentadult age range (17 years- 80 years), that is, all respondents who fit the DSM-IV criteria for schizophrenia or psychotic disorders (not for substance use disorders) as determined by the respondent's medical records and the results of consultations by the psychiatrist and the condition of the study's respondents who were stable (did not undergo inpatient psychiatric disorders in the last two months).

3) Type of treatment

The treatment is given to respondents in the form of social skills training therapy with a combination of social cognitive treatment therapy, both as an independent treatment or in combination with other therapies.

4) Measured research results

The results of the study measured were to assess the ability of patient outreach with others and social functions in general

\section{b. Exclusion criteria respondents}

Characteristics of research not included in this study are the types of research conducted on schizophrenia or psychosis respondents due to substance abuse, using psychopharmaceuticals in treatment delivery, community-based research, and testing of intervention models that are being developed

\section{Literature Search Strategy}

This systematic review was conducted by searching publication articles in the database: Pubmed, ScienceDirect, and Ebsco with the keywords Social cognitive intervention OR Social cognitive skills training AND schizophrenia OR psychotic disorder AND randomized control trial. Literature search strategy using the PICO method and making research questions.

Table 1. Table PICO

\begin{tabular}{cll}
\hline PICO & \multicolumn{2}{c}{ Detail } \\
\hline $\mathrm{P}$ & Patient schizophrenia & \\
\hline $\mathrm{I}$ & Social skill training & \\
\hline $\mathrm{C}$ & Control group & \\
\hline $\mathrm{O}$ & $\begin{array}{c}\text { socialization abilities } \\
\text { functions }\end{array}$ & \\
& \multicolumn{2}{c}{ social } \\
\hline
\end{tabular}

\section{Study Quality Study Methods}

Articles that fit the criteria are then analyzed using the critical appraisal tool that is appropriate for the results of the RCT research. The research was conducted by one person, the reviewer using a critical appraisal skills program (CSAP). The analyzed data is then extracted and synthesized according to the purpose.

\section{Extract dataresearch}

All data derived from the literature must meet the research inclusion criteria. Furthermore, the review process is done one by one in a way arranged in a table adjusted to the provisions to facilitate the review process. The research table consists of the author's name and research topic, the year of study, the type of therapy given to the experimental and control groups, the treatment method, the duration and frequency of therapy, and the measurement results. The extraction process is carried out by one person, the reviewer.

\section{Data synthesis}

Systematic Review is based on the characteristics of the study with inclusion criteria, namely 1) studies in schizophrenic patients in the adolescent-adult age range (17 years-80 years) (all patients who meet the DSM-IV criteria for schizophrenia or psychosis (not for substance use disorders) as determined by medical records and consultation by a psychiatrist as well as 
clinically stable subjects (there were no psychiatric inpatients in the last 2 months). 2) Interventions given must consist of social therapy skills training in a combination of social cognitive therapy therapies, either as independent interventions or in combination with Other interventions, full text, articles for 20092019, English-speaking journalist, International journal. The process of selecting articles is determined according to inclusion criteria. Sources of information were obtained from databases namely Pubmed, Scopus, Science Direct and EBSCO. Search strategy methods for obtaining journals using Social cognitive intervention, Social-cognitive skills training and using Advance Search "AND" schizophrenia, psychotic disorder. The process of collecting data using data extraction methods with the PICO (Population, Intervention, Compare, Outcome) approach. Articles are extracted from data in the form of citation, experimentation and control conditions, methods, duration and frequency, target domains, measurements, and final results. The method used to criticize journal articles is used using the Critical Appraisal Skills Program (CASP) instrument, this instrument identifies the literature through Screening questions. The method is to mitigate bias in this systematic review study. The results of the synthesis presented in this systematic review study consisted of the process of research identification, the screening process (filtration) of research results, the discovery of data in accordance with the research topic and the collection of data in accordance with the specified research inclusion criteria. Presentation of data is accompanied by the following flow diagram.

\section{RESULT}

\section{Characteristic of the research}

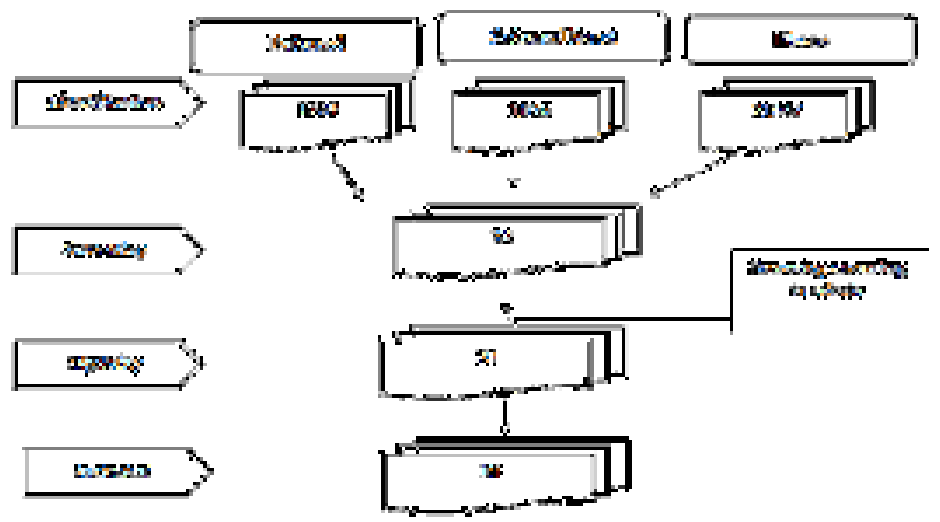

Figure 1. Flow Chart of the Review

Process.

The results of identification from the search method in Pubmed, the articles obtained were 1739 articles while the search with ScienceDirect obtained 1026 articles and searches with Ebsco as many as 21647 articles. Researchers conducted a screening including the results of the same type of search data and have the same research title. After the screening stage, the Eligibility stage is carried out, namely the suitability of the search data with the inclusion criteria set by the researchers including RCT research, or Quasiexperimental, schizophrenic respondents in the adolescent-adult age range (17 years- 80 years), the intervention given must consist from social therapy training in a combination of social cognitive treatment therapies, both as independent interventions or in combination with other interventions, the results obtained are minimal improvement in the ability of socialization and social functions in general, the literature in 2009-2019, English, and international journals. The results of screening to eligibility obtained literature data including 15 literature. The study results obtained a lot of results and carried 
out screening until eligibility to get documents that match the study criteria. Based on the results of the synthesis obtained 15 documents that fit the study criteria and then in-depth analysis (critical thinking) to get the best evidence of social therapy intervention training in schizophrenia. The quality of the articles reviewed has a minimum level of evidencebased practice (EBP) IIb, namely RCTs. RCTs are the highest quality research for experimental research. The results of the research literature have high credibility in the systematic process of research and the results are to be trusted.

Research results from(Reichenberg et al., 2009)" is an experimental randomized trial study. This study used a sample of 68 outpatients with random primary psychotic disorders who received 16 Social Cognitive Skills Training (SCST) therapies, 19 computerized neurocognitive remediation (NR), 19 standard illness management skills training (ST) people and A total of 14 patients were given Hybrid SCST and NR therapy. Patients are able to communicate and understand well. SCST therapy manages the patient's basic emotions, NR therapy is focused on ongoing attention, processing speed, and inhibition of response, ST therapy focused on Self-Management. The results showed SCST showed a greater improvement over time than NR $(Z=2.57, p=0.01)$ and ST $(Z=1.96, p=0.05)$. This intervention procedure can be applied to schizophrenia patients in Indonesia. This research has an EBP level, Ib.

(Horan et al., 2009)with the research title "Social cognitive skills training in schizophrenia: An initial efficacy study of stabilized outpatients" is an experimental study with a sampling method using random sampling. The sample in this study amounted to 34 randomly selected patients who were divided into case and control groups. Outpatients were recruited from the VA Los Angeles Health Center. Subjects were clinically stable (there were no psychiatric hospitalizations in the past 6 months, same antipsychotic medication for the past 3 months). Exclusion criteria in this study were past neurological disorders (eg, epilepsy), mental retardation, or drug use disorders in the past month. As many as 15 people received SCST therapy and as many as 16 control groups received disease selfmanagement intervention and recurrence prevention skills training. The results showed the results support the efficacy of a social cognitive intervention for dwelling outpatients and encourage further development of this treatment approach to achieve broader improvements in social cognition and generalization of treatment gains. This study has an Ib level.

(Rus-Calafell, GutiérrezMaldonado, Ortega-Bravo, Ribas-Sabaté, \& Caqueo-Urízar, 2013)Mar Rus et al. (2013) research is an experimental study with randomly. This study had a total sample of 31 divided into case and control groups, of which 13 participants received SST interventions and 18 participants as control groups and received treatment as usual (case management, medication adherence, psychotherapy, involvement, and family support). The results showed there was significance between the case group and the SST control group significantly improved from pre-to post-treatment $(\mathrm{t}=.65 .692, \mathrm{p}=$ $0.000)$. This intervention procedure can be applied to schizophrenia patients in Indonesia. This research has an EBP level, Ib.

Research conducted by (Kurtz, Mueser, Thime, Corbera, \& Wexler, 2015)with the research title "Social skills training and computer-assisted cognitive remediation in schizophrenia" is an 
experimental study with randomly. This study has a sample size of 89 respondents. The number of samples issued at the time of the study was 25 participants so that the remaining number of samples chosen at random were 64 participants divided into case and control groups. Case groups will be given Cognitive remediation interventions and 32 control group patients will be given Social-skills training interventions. The results showed the therapeutic group (COG REM) increased significantly (attention, working memory, and empathy) compared to the control group (Computer Skills). On the QLS-B scale it shows that individuals in the REM COG group increased more in empathy than in the Computer Skills group (F [1.45] $=7.13, \mathrm{p}=.011 ; \mathrm{d}=0.67)$. This intervention procedure can be applied to schizophrenia patients in Indonesia. This research has an EBP level, Ib.

Research by(Granholm, Holden, Link, \& McQuaid, 2014) with the title "Randomized Clinical Trial of Cognitive Behavioral Social Skills Training for Schizophrenia: Improvement in Functioning and Experiential Negative Symptoms" is a randomized clinical trial (RCT) study with randomly. A total of 149 samples were divided into case and control groups. As many as 74 people in the experimental group were given cognitive behavioral social skills training (CBSST) and 75 control groups were given goalfocused supportive contact (GFSC) therapy. The results showed that the Rating Scale for Psychosis (CTS-Psy) between cognitive behavioral social skills training (CBSST) $(\mathrm{M}=40.4, \mathrm{SD}=4.0)$ was significantly more significant than GFSC ( $M=19.7$, SD $=1.8), \mathrm{t}(22)=13.14, \mathrm{p}<.001$. CBSST therapy has proven to be more effective than GFSC. This research has an EBP level, $\mathrm{Ib}$.
Research conducted by (Bucci et al., 2013)with the title "Combining social cognitive treatment, cognitive remediation, and functional skills training in schizophrenia: a randomized controlled trial is an experimental study with randomly. The number of samples at the time of the assessment was 111 people. As many as 7 people were not included in the inclusion criteria, so 104 samples had to be removed that would be divided into cases and controls. A total of 52 people were given cognitive remediation group therapy (REHACOP) and 52 people were included in the control group. At the time of followup, there were 3 people who were excluded because they did not want to continue therapy at the time of the analysis of the number of control groups remaining as many as 49 people and were given occupational activities. The results showed that social relations were significant with value $(\mathrm{F}=8.11, \mathrm{P}=0.005)$. The combination of cognitive improvement, social cognitive intervention, and functional skills training shows statistically and clinically significant changes in neurocognition, social cognition, negative disability, and functional. This study has an EBP level, Ib

Research by (Rus-Calafell, Gutiérrez-Maldonado, \& Ribas-Sabaté, 2014)with the research topic "A virtual reality-integrated program for improving social skills in patients with schizophrenia: A pilot study" is a type of experimental research ". The number of samples at the time of the study was 15 people. As many as 3 people were not included in the inclusion criteria and so had to be excluded, the remaining 12 people would be divided into 6 groups of cases and controls. They are providing social skills training (SST) therapy to intervention groups that focus on social perception, social relations, assertive 
communication, role skills, and communication skills. The results showed negative symptoms, there was an improvement from pre-to post-treatment with an average decrease of $7.083(\mathrm{p}=$ 0.000 ), and in psychopathology, there was a decrease in the average of $9.33(p=0.001)$ there was a significant increase in negative symptoms psychopathology and social avoidance. This study has an EBP level, IIa.

Research conducted by (Kurtz et al., 2015) with the research topic "Social Skills Training and Computer-Assisted Cognitive Remediation in Schizophrenia" is experimental research with randomly. The number of samples at the time of the study was 89 people. As many as 25 people were not included in the inclusion criteria and thus had to be excluded, the remaining 64 samples would be divided into case and control groups with 32 people each. There are 3 types of therapy given to the case group: Social Skills Training, Cognitive Remediation, Control Computer Skills Condition. The results revealed the results showed that the Cognitive Remediation group increased significantly more in attention, working memory, and empathy than the Computer Skills group, but there were no differences between groups on other measures of function or psychosocial skills. Overall, these findings indicate that cognitive remediation used in the context of other evidence-based psychosocial interventions (SST) improves working memory in schizophrenia and suggests that this effect can be generalized to increase empathy. This study has an EBP level, Ib

Research by (Bowie, McGurk, Mausbach, Patterson, \& Harvey, 2012)with the research topic "Combined Cognitive Remediation and Functional Skills Training: Effects on Cognition, Functional Competence, and Real-World Behavior" is a type of experimental research with random sampling techniques. The number of samples at the time of the assessment was 119 people. As many as 5 people were not included in the inclusion criteria so they had to be excluded, there were 114 samples left to be divided into 3 groups of 29 cognitive remediation treatments, 28 people had functional adaptation skills training and 26 people had cognitive remediation of functional adaptation skills training. The results showed neurocognition to increase with long lasting effects after cognitive improvement. Social competence increases both with functional skills training and with combined cognitive remediation treatments alone do not produce significant improvements in real-world behavior. When combined with functional skills training statistically significant improve. This research has an EBP level, Ib.

Research by (Iwata et al., 2017) with the research title "Efficacy of cognitive rehabilitation using computer software with individuals living with schizophrenia: A randomized controlled trial in Japan " is an experimental study with randomly. Sixty-five patients with schizophrenia were randomly divided into training groups $(\mathrm{n}=33)$ or non-training groups $(\mathrm{n}=32)$, and compared executive performance with healthy controls $(n=33)$. The healthy control group (HCG) consisted of 33 people ( 16 women and 17 men). The results showed no significant effect of cognitive training on processing speed. Cognitive training in schizophrenic patients is effective for improving several aspects of executive function, but does not increase processing speed. This research has an EBP level, Ib.

Research conducted by (Granholm, Holden, Link, McQuaid, \& Jeste, 2013)with the research title Randomized Controlled Trial of Cognitive Behavioral Social Skills Training for Older Consumers With Schizophrenia: Defeatist Performance Attitudes and Functional Outcomes "is a 
randomized clinical trial (RCT) study with randomly. Respondents consisted of Veterans and non-veterans with schizophrenia or schizoaffective disorder in a number of 79 people with an age range of 45-78 years. The experimental group received 41 CBSST therapies and the control group received 38 Goal-Focused Supportive Contact (GFSC). The results showed CBSST is an effective treatment to improve functioning in older consumers with schizophrenia, and both CBS and other supportive goal-focused interventions can reduce symptoms of distress, increase motivation and self-esteem, and improve life satisfaction. This research has an EBP level, Ib.

The research entitled "The Effects of Metacognition-Oriented Social Skills Training on Psychosocial Outcome in Schizophrenia-Spectrum Disorders" (Inchausti et al., 2018) is a randomized clinical trial (RCT) with randomly. Participants numbered 69 patients who received psychosocial rehabilitation. Patients with 36 metacognition-oriented social skills training (MOSST) interventions 33 patients with standard intervention SST interventions. The results showed that group differences were significant in favor of MOSST on the Social and Occupational Functioning Assessment Scale (SOFAS) and Personal and Social Performance Scale (PSP) total scores at post-treatment follow-up. Concerning PSP subscales, there were significant between-group differences in favor of MOSST. This research has an EBP level, Ib.

Research by Eric (Granholm et al., 2014)with the topic Improvement in Negative Symptoms and Functioning in Cognitive-Behavioral Social Skills Training for Schizophrenia: Mediation by Defeatist Performance Attitudes and Asocial Beliefs "is a randomized clinical trial (RCT) study with randomly. Study sample with a diagnosis of schizophrenia $(\mathrm{N}=117)$ and schizoaffective disorder $(\mathrm{N}=32)$. The intervention group received a CognitiveBehavioral Social Skills Training (CBSST) therapy. The control group received the usual goal-focused supportive contact (GFSC) intervention. The findings suggest that cognitive-behavioral therapy interventions that target dysfunctional attitudes can lead to an improvement in negative symptoms and functioning in schizophrenia. The effects of CBSST on lower diminished motivation were also significantly mediated by lower ABS, as this mediation effect was statistically significant $(\mathrm{ab}=.00 .024, \mathrm{P}<.05)$. This research has an EBP level, Ib.

The study titled "A pilot study of social cognition and interaction training (SCIT) for schizophrenia " conducted by(Penn et al., 2005) is a controlled experimental study. The number of respondents was 10 respondents who received SoCIAL intervention as many as 5 people and received Social Skills And Neurocognitive Individualized Training (SSANIT) therapy of 5 people. The results showed a significant treatment effect (effect size: Cohen's $d$ 0.32) on the primary outcome; only the SoCIAL intervention improved theory of mind. Patients receiving SoCIAL intervention also showed an improvement of avolition. This study has an EBP level, IIa.

Furthermore, the research conducted by (Rus-Calafell et al., 2013) with the research title "A brief cognitivebehavioral social skills training for stabilized outpatients with schizophrenia: A preliminary study" is a type of experimental research with randomly. Thirty-one subjects completed the study. The experimental group received SST intervention of 13 people. The control group received 18 treatment-as-usual (TAU) interventions. Results support the efficacy of the SST brief for out patients with schizophrenia and show the need to implement empirically supported interventions in mental health services to enhance patients' 
social functioning and quality of life. This study has an EBP level, IIa.

\section{DISCUSSION}

The study results by collecting journal literature through systematic procedures and from accountable sources. The results of this Systematic Review by eliminating the results of the literature search to provide strict limits in the implementation of studies through inclusion criteria are 1) studies in schizophrenia patients in the adolescent-adult age range (17 years-80 years) (all patients who meet the DSM-IV criteria for schizophrenia or psychosis (not for substance use disorders) as determined by medical records and consultation by psychiatrists and stable clinical subjects (no psychiatric hospitalization in the past 2 months) 2) Interventions given must consist of social therapy skills training in combination with cognitive therapy therapy social studies, either as independent interventions or in combination with other interventions, studies have a minimum level of EBP Iib, full text, articles in 2009-2019, English language journals, international journals. Variables in research are social skill training (SST) or social skill intervention and schizophrenia. The study identified the process of selecting selected articles. Sources of information were obtained from databases namely Pubmed, Scopus, Science Direct and EBSCO. Search strategy methods for obtaining journals using Social cognitive intervention, Social-cognitive skills training and using Advance Search "AND" schizophrenia, psychotic disorder. The process of collecting data using data extraction methods with the PICO (Population, Intervention, Compare, Outcome) approach. Articles are extracted from data in the form of citation, experimentation and control conditions, methods, duration and frequency, target domains, measurements, and final results. The method used to criticize journal articles is used using the Critical Appraisal Skills
Program (CASP) instrument, this instrument identifies the literature through Screening questions. The method is to mitigate bias in this systematic review study. The forms of synthesis that will be presented in a systematic review are identification, screening, eligibility, and included.

The results of a systematic review study found that the combination of SST therapy combined with cognitive and behavioral treatment was significant to overcome social damage in schizophrenic patients. The therapy is able to manage basic emotions, attention, self-management that is good for patients. Combined SST therapy can also change social perceptions and judgments when interacting with others. The combination interventions given can also improve the quality of life of patients with schizophrenia in general. Through interpersonal relationship training, effective communication, rational thought processes without the presence of negative thoughts that are trained simultaneously will have a positive impact on patients. Patients are more accepted in the community because negative stigma about schizophrenia patients is reduced so that patients are able to be productive and minimize recurrence rates. Schizophrenia is one of the ten main causes of functional disability globally (Altamura, Fagiolini, Galderisi, Rocca, \& Rossi, 2014). It is possible to strengthen the psychosocial impact of interventions on negative symptoms by targeting dysfunctional attitudes. Beck and colleagues (2009) propose that cognitions such as relentless performance attitudes and asocial beliefs contribute to negative symptoms and poor functioning in schizophrenia. Beck proposes everyday experiences of failure (for example, poor grades in school, poor work performance, social rejection) because factors associated with illnesses such as neurocognitive disorders and stigma can cause low expectations for a successful attitude which in turn leads to negative 
symptoms like not motivated and shut down from the social environment. Likewise in social learning, trust and selfcompetence are central to motivation for achievement and involvement in positive functions directed at the objectives of the activity(Wenger, 1998).

Based on the results of the study found that the use of computer-based and manual combination SST therapy did not differ significantly. This is because combination SST therapy is observed only in speaking skills but not in vocal and nonverbal skills. It seems worth noting that speaking skills items reflect the social nature of reciprocal interactions (eg, length of response, use of social reinforcement, and turn taking) or social intelligence (ie behavior in public and certain situations). In contrast, vocal and nonverbal skill items include unidirectional traits in social interactions (Bellack et al., 2004;). In short, this study equally supports the efficacy and specificity of targeted social interventions for patients with psychosis. Limitations include administrative facilities and the absence of diagnostic interview standards, which can limit generalizations. In addition, participants receive compensation, which can affect the level of attendance, and the therapist's compliance with treatment protocols and the durability of the effects of training due to saturation should be noted. Despite the limitations and challenges of developing the treatment, current findings point to the potential benefits of social cognitive training for people with psychotic disorders (Morvan \& O’Connor, 2017).

The results of the data can be analyzed that social skills training (SST) interventions combined with cognitive and behavioral treatment can be applied to patients with schizophrenia in adolescent to adult groups. The strength of this study is that it has the same characteristics and is easily applied to the order of health services everywhere. Social skills training (SST) interventions combined with cognitive and behavioral treatment can be applied by health services by applying NCP to nursing care in patients with schizophrenia, so that improving health status in schizophrenic patients can be improved. Policy makers in the health service can also include these interventions in a program of activities that can be implemented together which ultimately leads to a healthy community.

\section{CLOSING}

Social skills training (SST) combined with cognitive and behavioral treatment is effective in increasing the socialization and social function of schizophrenic patients. This is evidenced by various studies that have been published internationally so that services to patients with schizophrenia can effectively make a healthy community as a whole both physically and mentally healthy. The results of this systematic review can be recommended to future researchers namely how the effectiveness of Social Skills Training (SST) combined with cognitive and behavioral treatment in community groups.

\section{REFFERENCES}

Altamura, C., Fagiolini, A., Galderisi, S., Rocca, P., \& Rossi, A. (2014).

Schizophrenia today: epidemiology, diagnosis, course and models of care La schizofrenia oggi: epidemiologia, diagnosi, decorso e modelli di cura. In Original article Journal of Psychopathology.

Bowie, C. R., McGurk, S. R., Mausbach, B., Patterson, T. L., \& Harvey, P. D. (2012). Combined cognitive remediation and functional skills training for schizophrenia: Effects on cognition, functional competence, and real-world behavior. American

Journal of Psychiatry. https://doi.org/10.1176/appi.ajp.2012. 11091337 
Bucci, P., Piegari, G., Mucci, A., Merlotti, E., Chieffi, M., De Riso, F., ... Galderisi, S. (2013). Neurocognitive individualized training versus social skills individualized training: A randomized trial in patients with schizophrenia. Schizophrenia Research.

https://doi.org/10.1016/j.schres.2013.0 7.053

Burns, J. (2006). The social brain hypothesis of schizophrenia. Psychiatria Danubina.

Chavushyan, A., Hovsepyan, M., \& Boyajyan, A. (2013). Cryoglobulins as Potential Triggers of Inflammation in Schizophrenia. Schizophrenia Research and Treatment. https://doi.org/10.1155/2013/125264

Granholm, E., Holden, J., Link, P. C., \& McQuaid, J. R. (2014). A randomized clinical trial of cognitive behavioral social skills training for schizophrenia: Improvement in the functioning and experiential negative symptoms. Journal of Consulting and Clinical Psychology. https://doi.org/10.1037/a0037098

Granholm, E., Holden, J., Link, P. C., McQuaid, J. R., \& Jeste, D. V. (2013). Randomized controlled trial of cognitive behavioral social skills training for older consumers with schizophrenia: Defeatist performance attitudes and functional outcome. American Journal of Geriatric Psychiatry.

https://doi.org/10.1016/j.jagp.2012.10. 014

Green, M. F., Olivier, B., Crawley, J. N., Penn, D. L., \& Silverstein, S. (2005). Social cognition in schizophrenia: Recommendations from the Measurement and Treatment Research to Improve Cognition in Schizophrenia New Approaches Conference. Schizophrenia Bulletin. https://doi.org/10.1093/schbul/sbi049

Horan, W. P., Kern, R. S., Shokat-Fadai,
K., Sergi, M. J., Wynn, J. K., \& Green, M. F. (2009). Social cognitive skills training in schizophrenia: An initial efficacy study of stabilized outpatients. Schizophrenia Research. https://doi.org/10.1016/j.schres.2008.0 9.006

Inchausti, F., García-Poveda, N. V., Ballesteros-Prados, A., Ortuño-Sierra, J., Sánchez-Reales, S., Prado-Abril, J., ... Fonseca-Pedrero, E. (2018). The effects of metacognition-oriented social skills training on psychosocial outcome in schizophrenia-spectrum disorders: A randomized controlled trial. Schizophrenia Bulletin. https://doi.org/10.1093/schbul/sbx168

Iwata, K., Matsuda, Y., Sato, S., Furukawa, S., Watanabe, Y., Hatsuse, N., \& Ikebuchi, E. (2017). Efficacy of cognitive rehabilitation using computer software with individuals living with schizophrenia: A randomized controlled trial in Japan. Psychiatric Rehabilitation Journal. https://doi.org/10.1037/prj0000232

Kurtz, M. M., Mueser, K. T., Thime, W. R., Corbera, S., \& Wexler, B. E. (2015). Social skills training and computerassisted cognitive remediation in schizophrenia. Schizophrenia Research. https://doi.org/10.1016/j.schres.2015.0 1.020

Morvan, C., \& O'Connor, A. J. (2017). A theory of cognitive dissonance. In $A$ Theory of Cognitive Dissonance. https://doi.org/10.4324/978191228243 2

Mudge, J., Miller, N. A., Khrebtukova, I., Lindquist, I. E., May, G. D., Huntley, J. J., ... Kingsmore, S. F. (2008). Genomic convergence analysis of schizoprenia: mRNA sequencing reveals altered synaptic vesicular transport in post-mortem cerebellum. PLOS ONE. https://doi.org/10.1371/journal.pone.0 003625 
Penn, D., Roberts, D. L., Munt, E. D., Silverstein, E., Jones, N., \& Sheitman, B. (2005). A pilot study of social cognition and interaction training (SCIT) for schizophrenia [1]. Schizophrenia Research. https://doi.org/10.1016/j.schres.2005.0 7.011

Reichenberg, A., Harvey, P. D., Bowie, C. R., Mojtabai, R., Rabinowitz, J., Heaton, R. K., \& Bromet, E. (2009). Neuropsychological function and dysfunction in schizophrenia and psychotic affective disorders. Schizophrenia Bulletin. https://doi.org/10.1093/schbul/sbn044

Rus-Calafell, M., Gutiérrez-Maldonado, J., Ortega-Bravo, M., Ribas-Sabaté, J., \& Caqueo-Urízar, A. (2013). A brief cognitive-behavioural social skills training for stabilised outpatients with schizophrenia: A preliminary study.

Schizophrenia Research. https://doi.org/10.1016/j.schres.2012.1 1.014

Rus-Calafell, M., Gutiérrez-Maldonado, J., \& Ribas-Sabaté, J. (2014). A virtual reality-integrated program for improving social skills in patients with schizophrenia: A pilot study. Journal of Behavior Therapy and Experimental Psychiatry. https://doi.org/10.1016/j.jbtep.2013.09 .002

Saito, N., Kuginuki, T., Yagyu, T., Kinoshita, T., Koenig, T., PascualMarqui, R. D., ... Lehmann, D. (1998). Global, regional, and local measures of complexity of multichannel electroencephalography in acute, neuroleptic-naive, first-break schizophrenics. Biological Psychiatry. https://doi.org/10.1016/S00063223(97)00547-7

Tényi, T., Herold, R., Szili, I. M., \& Trixler, M. (2002). Schizophrenics show a failure in the decoding of violations of conversational implicatures. Psychopathology. https://doi.org/10.1159/000056212

Verdoux, H., \& Van Os, J. (2002).

Psychotic symptoms in non-clinical populations and the continuum of psychosis. Schizophrenia Research. https://doi.org/10.1016/S09209964(01)00352-8

Weisz, J. R., Sandler, I. N., Durlak, J. A., \& Anton, B. S. (2005). Promoting and protecting youth mental health through evidence-based prevention and treatment. American Psychologist. https://doi.org/10.1037/0003066X.60.6.628

Wenger, E. (1998). Communities of Practice: Learning, Meaning, and Identity. Systems Thinker. https://doi.org/10.2277/0521663636

Witthöft, M., \& Jasper, F. (2015). Somatic Symptom Disorder. In Encyclopedia of Mental Health: Second Edition. https://doi.org/10.1016/B978-0-12397045-9.00096-3 